\title{
Apalutamide/Leuprolide Regimen
}

National Cancer Institute

\section{Source}

National Cancer Institute. Apalutamide/Leuprolide Regimen. NCI Thesaurus. Code C160520.

A regimen consisting of apalutamide and leuprolide that can be used for the treatment of prostate cancer. 